\title{
Pérdidas productivas y económicas diarias ocasionadas por la mastitis y erogaciones derivadas de su control en establecimientos lecheros de Córdoba, Argentina ${ }^{\#}$
}

\author{
Productive and economic daily losses due to mastitis and its control expenditures \\ in dairy farms in Córdoba, Argentina \\ C Vissio $^{a^{*}}$, DA Agüero ${ }^{a}$, CG Raspanti ${ }^{b}$, LM Odierno $^{b}$, AJ Larriestra ${ }^{a}$ \\ aFacultad de Agronomía y Veterinaria, Universidad Nacional de Río Cuarto, Córdoba, Argentina. \\ bFacultad de Ciencias Exactas, Físicas-Químicas y Naturales, Universidad Nacional de Río Cuarto, Córdoba, Argentina.
}

\begin{abstract}
SUMMARY
The aim was estimate the daily direct cost and the expenditures due to mastitis control and prevention, evaluating their magnitude and variation among dairy farms. A random sample of dairy farms was the study population. Forty eight farms were visited once. The visit included a clinical mastitis check-up of lactating cows, a visual inspection of milking routine, and the analysis of a questionnaire. Milk composite samples $(\mathrm{n}=1955)$ were randomly collected for somatic cell count (SCC). Productive and economic daily losses were characterised and quantified according to the composite sample SCC and valuing discarded milk as result of the clinical onset. Dairy farm control and prevention expenditures were also determined. The losses for subclinical mastitis showed a median of 2.8 liters/milking cow/day (percentile $25 \%=2.0$; percentile $75 \%=2.9$ ) for all cows studied, that represents US\$ 0.99/milking cow/day. Losses due to milk discarded for clinical mastitis, showed a dairy farms median 0.12 liters/milking cow/day (percentile $25 \%=0$; percentile $75 \%=0.31$ ), that represents a cost of US $\$ 0.04 /$ milking cow/day. The control and prevention expenditures dairy farm median was about US $\$ 0.06 /$ milking cow/day (percentile $25 \%=0.034$; percentile $75 \%=0.095$ ). The total cost of mastitis, including the loss caused by the disease and the cost of its control and prevention measures were $\geq$ US\$ 1.0/milking cow/day (percentile $25 \%=0.8$; percentile $75 \%=1.2$ ) in at least $50 \%$ of the dairy farms. Daily total cost for mastitis represented $16 \%$ of the dairy farm daily gross income in at least $50 \%$ of the dairies. No difference of cost across distinct farm production strata was found, except by the ratio of total mastitis cost over daily gross income. That ratio was higher among low producing farms. This is the first report about mastitis costs variation among small and medium dairy farms in Argentina.
\end{abstract}

Key words: bovine mastitis, economic impact, mastitis total cost, mastitis direct losses.

\section{RESUMEN}

El propósito de este estudio fue estimar los costos diarios directos de la mastitis y las erogaciones derivadas de su control y prevención, y describir su magnitud y variación entre establecimientos lecheros de una de las principales cuencas lecheras de Argentina. Una muestra aleatoria de establecimientos lecheros $(\mathrm{n}=48)$ fueron visitados una sola vez. En todas las vacas en ordeño se verificó la presencia de mastitis clínica $(\mathrm{MC})$ y a 1.955 vacas seleccionadas aleatoriamente se les extrajo una muestra compuesta de leche para determinación de recuento celular somático (RCS). Simultáneamente se examinó la rutina de ordeño y se analizó una encuesta aplicada al encargado de ordeño. Se caracterizaron y cuantificaron las pérdidas productivas y económicas de acuerdo con el RCS, en concepto de leche descartada por MC y las erogaciones por acciones de control y prevención. La mediana de pérdidas directas invisibles fue de 2,8 litros/vaca/día (percentil $25 \%=2,0$; percentil $75 \%=2,9$ ) para el conjunto de animales en estudio, representando un costo de US\$ 0,99/vaca/día. La mediana de pérdidas en el establecimiento atribuidas a MC fue de 0,12 litros/vaca/día (percentil 25\% $=0 ;$ percentil 75\% $=0,31$ ), representando un costo de US\$ 0,04/vaca/día. La mediana de pérdidas indirectas fue de US\$ 0,06/vaca/día (percentil 25\% = 0,034; percentil 75\% $=$ 0,095). En el $50 \%$ de los establecimientos el costo total, es decir, adicionando las pérdidas en producción de leche a los gastos del control y prevención fue $\geq$ US $\$ 1,0 / \mathrm{vaca} /$ día (percentil $25 \%=0,8$; percentil $75 \%=1,2$ ). El costo total de la mastitis, representó en promedio $16 \%$ de los ingresos brutos diarios en al menos la mitad de los establecimientos. Al analizar los establecimientos de acuerdo con tres niveles de producción, no se observaron diferentes perfiles de costos; con excepción de la relación costos sobre ingresos brutos, la que fue mayor en el estrato de menor producción. Este es el primer trabajo que explora diferencias de costos de la mastitis en una muestra aleatoria de productores lecheros de Argentina.

Palabras clave: mastitis bovina, impacto económico, costo total de la mastitis, pérdidas directas por mastitis.

\section{INTRODUCCIÓN}

El objetivo principal del análisis económico de una enfermedad es brindar un conocimiento detallado de sus

Aceptado: 19.06.2014.

\# Financiado por SECyT (Secretaría de Ciencia y Técnica, Universidad Nacional de Río Cuarto) y FONCyT (Agencia Nacional de Promoción Científica y Tecnológica).

* Ruta Nac. 36 - Km. 601 - Código Postal X5804BYA Río Cuarto, Córdoba, Argentina; cvissio@ayv.unrc.edu.ar costos asociados (Hogeveen y col 2011). En el caso de la mastitis bovina, las pérdidas productivas debidas a casos clínicos son apreciables con facilidad por el productor, al contrario de lo que sucede con aquellas derivadas de la manifestación subclínica de la enfermedad (Van Asseldonk y col 2010). Las pérdidas asociadas a la mastitis subclínica (MS) son estimadas a partir del recuento de células somáticas (RCS) en leche, ya que existe una estrecha relación entre este, el nivel de infección intramamaria (IIM) y la magnitud de las pérdidas físicas (Bartlett y col 1990, Miller 
y col 1993b, Dürr y col 2008, Hagnestam-Nielsen y col 2009). En un establecimiento comercial, la estimación de pérdidas puede realizarse mediante muestras de tanque o por medio de la colecta de muestras de leche compuesta a nivel de vaca (Reneau y col 2001).

Los costos asociados a mastitis incluyen disminución en la producción y calidad de leche, leche descartada, gastos derivados de honorarios veterinarios, compra de drogas, trabajo extra, descarte de vacas y ocurrencia de enfermedades concomitantes (Schepers y Dijkhuizen 1991, Halasa y col 2007). Estos factores pueden explicar las variaciones de costos totales de la enfermedad e influir drásticamente en la conveniencia o no del control de la misma a nivel predial (McInnerney y col 1992).

Las pérdidas por producción atribuidas a la mastitis están relacionadas a sus formas de presentación, clínica y subclínica, las que afectan en diferente grado el nivel de producción (Seegers y col 2003), el riesgo de descarte (Seegers y col 2003) o muerte de la vaca (Miller y col 1993a) y las chances de fallas reproductivas (Lavon y col 2011). Una parte importante de las pérdidas asociadas a la mastitis podrían deberse a la forma subclínica de la enfermedad, como fue documentado en los trabajos de Yalcin (2000) y Huijps y col (2008). Asimismo, las pérdidas por descarte prematuro y otras derivadas de la forma clínica de presentación han sido reportadas como las más relevantes (Halasa y col 2007, Hagnestam-Nielsen y Ǿstergaard 2009). La comparación entre estudios se ve dificultada, entre otras razones, por las diferencias en la forma de estimación de las pérdidas (Seeger y col 2003, Halasa y col 2007). En algunos casos, las pérdidas han sido valoradas a partir de la determinación del RCS en tanque y de la incidencia de MC (Miller y col 1993a, Geary y col 2012), y en otros, sistematizando la percepción de costos por parte del productor (Huijps y col 2010) o mediante modelos de simulación (Halasa y col 2009a).

Además de considerar el efecto productivo de la enfermedad, el análisis económico involucra la valoración de las acciones de control y prevención (Rushton 2009, Hogeveen y col 2011). Según McInnerney (1992), existe una relación de substitución entre las pérdidas por mastitis y las erogaciones derivadas de su control, donde el óptimo de control de la enfermedad es aquel que minimiza el costo total, es decir, que incluye las pérdidas por producción más las erogaciones de su control y prevención.

Gran parte del entendimiento de los costos asociados a la mastitis proviene de estudios realizados en el hemisferio norte, tal como ha sido documentado en las revisiones de Seegers y col (2003), Halasa y col (2007) y Hogeveen y col (2011), siendo escasos los reportes con datos de origen latinoamericano (Graaf y Dwinger 1996, Romero y col 2004, Pech Martínez y col 2007, Bedolla y Ponce de Leon 2008). Adicionalmente, las particularidades de los sectores lecheros nacionales y sus respectivos precios relativos limita la comparación entre estudios y la posibilidad de establecer patrones de referencia.
En Argentina no se dispone de estadísticas actualizadas acerca de las pérdidas económicas causadas por la mastitis (Calvinho y Tirante 2005). Las últimas estimaciones, realizadas hace tres décadas, indican pérdidas anuales por menor producción de US\$ 115 a US\$ 220 millones por año (González y col 1977, Asociación Argentina de Lucha Contra Mastitis 1983). Además, no existen precedentes de investigaciones dirigidas a examinar las pérdidas directas y las erogaciones de control y prevención de esta enfermedad, y observar la variabilidad entre establecimientos. El objetivo de este estudio fue estimar los costos diarios directos asociados a la presencia de mastitis y las erogaciones derivadas de su control y prevención, además, describir la variación entre establecimientos lecheros de Córdoba, una de las principales provincias productoras de leche de Argentina.

\section{MATERIAL Y MÉTODOS}

\section{SELECCIÓN DE LOS ESTABLECIMIENTOS LECHEROS}

La muestra estudiada incluyó 48 establecimientos lecheros pertenecientes a la Unidad Ejecutora Local (UEL) Villa María, Córdoba, Argentina. La selección se realizó en forma simple y al azar a partir de un universo de establecimientos lecheros que poseían entre 100 a 250 vacas totales $(\mathrm{N}=338)$, dicho estrato representa el $65 \%$ de los productores de Argentina (Castellanos y col 2009). Los establecimientos seleccionados en el estudio fueron visitados solo una vez durante el ordeño entre los meses de marzo y septiembre de 2007.

\section{SELECCIÓN DE ANIMALES}

La cantidad de vacas seleccionadas en cada establecimiento fue calculada considerando una prevalencia de IIM del $20 \%$, una precisión absoluta del $10 \%$ y una confianza del 95\%, utilizando las ecuaciones descritas por Thrusfield (2007) e implementada en el programa EPIDAT 3.1 (Xunta de Galicia, OPS. 2006). Las vacas incluidas en el estudio fueron elegidas en cada establecimiento mediante un procedimiento de selección al azar sistemático. Para esto, el total de vacas en ordeño se dividió por el tamaño de la muestra establecido previamente. De esta manera se identificó el intervalo de selección, para posteriormente elegir al azar la primera vaca (Thrusfield 2007). A los animales seleccionados se les extrajo una muestra compuesta de leche, esto es de los cuatro cuartos, y a partir de la identificación individual se registró el número ordinal de parto y los días en lactación. Cada muestra de leche fue obtenida de manera aséptica inmediatamente antes del ordeño. Luego de realizar el despunte o descarte de los primeros chorros de leche, se detectó la presencia de MC mediante la visualización de alteraciones físicas en la leche o en la ubre (NMC 2003). Luego de que los pezones con un algodón embebido en etanol $70 \%$ fueron 
desinfectados, un volumen aproximado de $50 \mathrm{ml}$ de una muestra compuesta de los 4 cuartos se recolectó en tubos de plástico estéril, conservándose las muestras a $4{ }^{\circ} \mathrm{C}$ y con azidiol hasta el arribo al laboratorio del Departamento de Microbiología de la Facultad de Ciencias Exactas, Fisico-Químicas y Naturales de la Universidad Nacional de Río Cuarto.

\section{RECUENTO CELULAR SOMÁTICO}

El RCS fue determinado mediante Somacount 300 (Bentley), dentro de las 24 horas posteriores a su recolección. Inicialmente la muestra de leche fue colocada en un baño María a $40^{\circ} \mathrm{C}$, luego homogeneizada con un agitador incorporado al equipo para la posterior determinación de RCS. Los resultados fueron expresados como cél $/ \mathrm{ml}$ y para su análisis fueron transformados a logaritmo natural (ln) (Ali y Shook 1980).

\section{RECOLECCIÓN DE DATOS}

La información de cada establecimiento utilizada en el presente trabajo fue generada en el estudio de Vissio y col (2013). Este implicó el examen visual de la rutina de ordeño y la administración de un cuestionario sobre el manejo de la salud mamaria a la persona encargada del ordeño. Las prácticas de control y prevención consideradas en este análisis fueron: desinfección o sellado postordeño de pezones, tratamiento antibiótico de casos de MC y tratamiento antibiótico masivo al momento del secado. Se realizó una valoración económica de cada práctica considerando que se tenía registro del producto utilizado y la forma de administración.

\section{PÉRDIDAS FÍSICAS POR MASTITIS}

Las pérdidas de leche diarias debido a MS fueron deducidas a partir del RCS de cada vaca muestreada, según la siguiente ecuación propuesta por Dürr y col (2008),

$$
\mathrm{PDL}_{\mathrm{MS}}=(\ln (\mathrm{RCS} / 1000)-2) * \mathrm{RPD}
$$

donde, $\mathrm{PDL}_{\mathrm{MS}}$ es la pérdida diaria de leche por MS/vaca, RCS es el actual recuento de células somáticas, -2 es el punto de corte en escala logarítmica donde comienzan las pérdidas de leche (equivalente a aproximadamente 7.400 cél $/ \mathrm{ml}$ ) y RPD es la pérdida esperada de leche por unidad de incremento en $\operatorname{lnRCS}$, de acuerdo con su raza $(\mathrm{R})$, número de parto (P) y los días en lactación (D) (Dürr y col 2008).

Las pérdidas en producción de leche debidas a MS fueron estimadas mediante el cálculo del intervalo de confianza, ya que se trabajó con una muestra aleatoria de vacas por establecimiento (Thrusfield 2007). En esta investigación el valor del límite inferior del intervalo de confianza fue elegido para realizar las estimaciones de pérdidas por MS y pérdidas totales en cada establecimiento.
Las pérdidas físicas por $\mathrm{MC}$ fueron estimadas considerando el número de vacas con $\mathrm{MC}$ el día de la visita y el promedio de producción por vaca en cada establecimiento. La fórmula utilizada fue la siguiente,

$$
\mathrm{PDL}_{\mathrm{MC}}=\mathrm{VMC} * \mathrm{LVD} / \mathrm{VO}
$$

donde, $\mathrm{PDL}_{\mathrm{MC}}$ es la pérdida diaria de leche (litros/vaca/ día) por MC, VMC es el número de vacas con mastitis clínica en el día de la visita al establecimiento, LVD es la producción promedio de leche por vaca y VO es el total de vacas en ordeño en el establecimiento. La producción promedio de leche por vaca fue calculada dividiendo el volumen en litros medido en el tanque de frío al final del ordeño por el número de vacas ordeñadas.

\section{PÉRDIDAS ECONÓMICAS POR MASTITIS}

La valoración económica de las pérdidas productivas fueron obtenidas mediante la multiplicación de las pérdidas físicas atribuidas a MS y MC por el precio de la leche, respectivamente. Las estimaciones fueron realizadas considerando un precio de mercado de 35 centavos de dólar (cotización del 12/02/2012). Además, todos los cálculos adicionales y comparaciones fueron llevados a moneda constante.

Las erogaciones derivadas de las acciones de control y prevención consideradas en este estudio fueron: desinfección postordeño de pezones, tratamiento antibiótico de casos de MC y terapia antibiótica masiva al momento del secado. El precio del desinfectante de pezones fue valorado en 0,007 centavos de dólar por vaca por día. Para estimar las erogaciones por la aplicación de terapia de secado se asumió: (1) una lactancia de 305 d, (2) un periodo de secado de 60 d y (3) una distribución uniforme a lo largo de todo el año. Esta información permitió determinar para cada establecimiento el número de vacas que son secadas por día y esto multiplicado por el precio del producto utilizado para la terapia al secado permitió estimar la erogación diaria por establecimiento; para obtener una estimación por vaca este valor fue dividido por el número de vacas en ordeño. Las erogaciones por tratamiento de casos de MC derivan de la cantidad de casos en cada establecimiento detectados en el día de la visita, multiplicado por el valor monetario de la aplicación y dividido por el total de vacas en ordeño.

El costo total de la mastitis en cada establecimiento fue obtenido mediante la sumatoria del valor en dólares correspondiente a las pérdidas productivas por MS y MC y las erogaciones por la aplicación de las prácticas de control y prevención. Complementariamente se estandarizó el costo total de la enfermedad en el establecimiento, dividiendo los costos por el ingreso bruto del establecimiento en el día de la visita y expresándose esta relación de forma porcentual. 


\section{RESULTADOS}

ESTABLECIMIENTOS ESTUDIADOS Y CARACTERÍSTICAS DE LOS ANIMALES

En los 48 establecimientos visitados el número promedio de vacas totales fue de 164 (rango 95-284), siendo 128 el valor promedio de vacas en ordeño por establecimiento (rango 70-212). La media de producción de leche por establecimiento fue de 2.231 litros/día, con un mínimo de 600 litros/día y un máximo de 4.300 litros/día. Un total de 1.955 vacas fueron muestreadas (promedio de 41 vacas por establecimiento, $\mathrm{DE}=5,7$ ). Los animales muestreados fueron de raza Holstein y tuvieron una mediana de días en lactación de 138 (percentil 25\% = 66; percentil $75 \%=236$ días) y $24 \%$ correspondió a vacas de primer parto.

En cuanto a la frecuencia de mastitis encontrada, el $1,0 \%(65 / 6.158)$ de las vacas mostró evidencias de la forma clínica de la enfermedad y 97,4\% (1.904/1.955) de los animales muestreados evidenciaron RCS superiores al umbral mínimo, a partir de ello se pueden deducir pérdidas de producción $(7.400 \mathrm{cél} / \mathrm{ml})$. La aplicación de las prácticas de control y prevención relevadas en este estudio fue variable entre los 48 establecimientos estudiados (cuadro 1).

\section{ESTIMACIÓN DE LAS PÉRDIDAS FÍSICAS Y ECONÓMICAS} POR MASTITIS

La mediana de pérdidas en producción de leche por MS fue de 2,8 litros/vaca/día (percentil $25 \%=2,0$; percentil $75 \%=2,9$ ), lo que representa un costo de US\$ $0,99 / \mathrm{vaca} /$ día (cuadro 2). Por otro lado, la mediana de pérdidas en producción de leche atribuidas a MC fue de 0,12 litros/ vaca/día (percentil 25\% =0,0; percentil 75\% =0,31), lo que representa un costo de US\$ 0,04/vaca/día (cuadro 2). Las estimaciones de pérdidas por MS y MC a nivel de establecimiento evidenciaron una gran disparidad entre los mismos (figura 1). Entre aquellos establecimientos con pérdidas económicas por MS superiores a la mediana, no se observó un patrón de mayores pérdidas por MC (figura 1). Por ejemplo, la mediana de pérdidas por MC en el 50\% de los establecimientos con mayores pérdidas por MS (figura 1, segmento derecho) fue de 6,72 (percentil $25 \%=0,00$; percentil $75 \%=16,23$ ) y en el $50 \%$ de los establecimientos con menores pérdidas por MS (figura 1, segmento izquierdo) fue de 5,43 (percentil 25\% = 0,00; percentil 75\% = 7,63).

\section{ESTIMACIÓN DE LAS EROGACIONES DERIVADAS DEL} CONTROL Y PREVENCIÓN

Para el conjunto de los establecimientos estudiados, la mediana de erogaciones derivados del control y prevención

Cuadro 1. Grado de aplicación de prácticas de control y prevención en establecimientos lecheros de Córdoba, Argentina ( $\mathrm{n}=48$ ). Degree of application of the control and prevention measures in dairy farms from Córdoba, Argentina $(n=48)$.

\begin{tabular}{lccc}
\hline $\mathrm{N}^{\circ}$ establecimientos $(\%)$ & Desinfección postordeño & Tratamiento MC & Terapia de secado \\
\hline $19(39,6)$ & & $\mathrm{X}$ & $\mathrm{X}$ \\
$18(37,5)$ & $\mathrm{X}$ & $\mathrm{X}$ & $\mathrm{X}$ \\
$8(16,7)$ & & $\mathrm{X}$ & \\
$2(4,2)$ & & $\mathrm{X}$ & \\
$1(2,1)$ & $\mathrm{X}$ & $\mathrm{X}$ & \\
\hline
\end{tabular}

Cuadro 2. Componentes del costo total de la mastitis expresado en dólares por día en establecimientos lecheros de Córdoba, Argentina $(\mathrm{n}=48)$.

Components of mastitis total cost (U\$S/day) in dairy farms from Córdoba, Argentina ( $\mathrm{n}=48)$.

\begin{tabular}{llll}
\hline & & $\begin{array}{c}\text { Mediana a nivel de vaca } \\
\text { (rango intercuartil) }\end{array}$ & $\begin{array}{c}\text { Mediana a nivel de establecimiento } \\
\text { (rango intercuartil) }\end{array}$ \\
\hline Costos directos & MC & $0,041(0,000-0,108)$ & $6,173(0,000-11,180)$ \\
& MS & $0,986(0,527-1,602)$ & $104,126(81,670-134,577)$ \\
Erogaciones por & Tratamiento de vacas con MC & $0,006(0,000-0,027)$ & $0,837(0,000-3,259)$ \\
& Terapia de secado & $0,047(0,014-0,054)$ & $4,643(2,134-7,630)$ \\
& Desinfección postordeño de pezones & $0,000(0,000-0,007)$ & $0,000(0,000-0,798)$ \\
Costos totales & Total & $0,059(0,034-0,095)$ & $6,887(4,097-11,697)$ \\
\hline
\end{tabular}

Fuente: elaboración propia. 


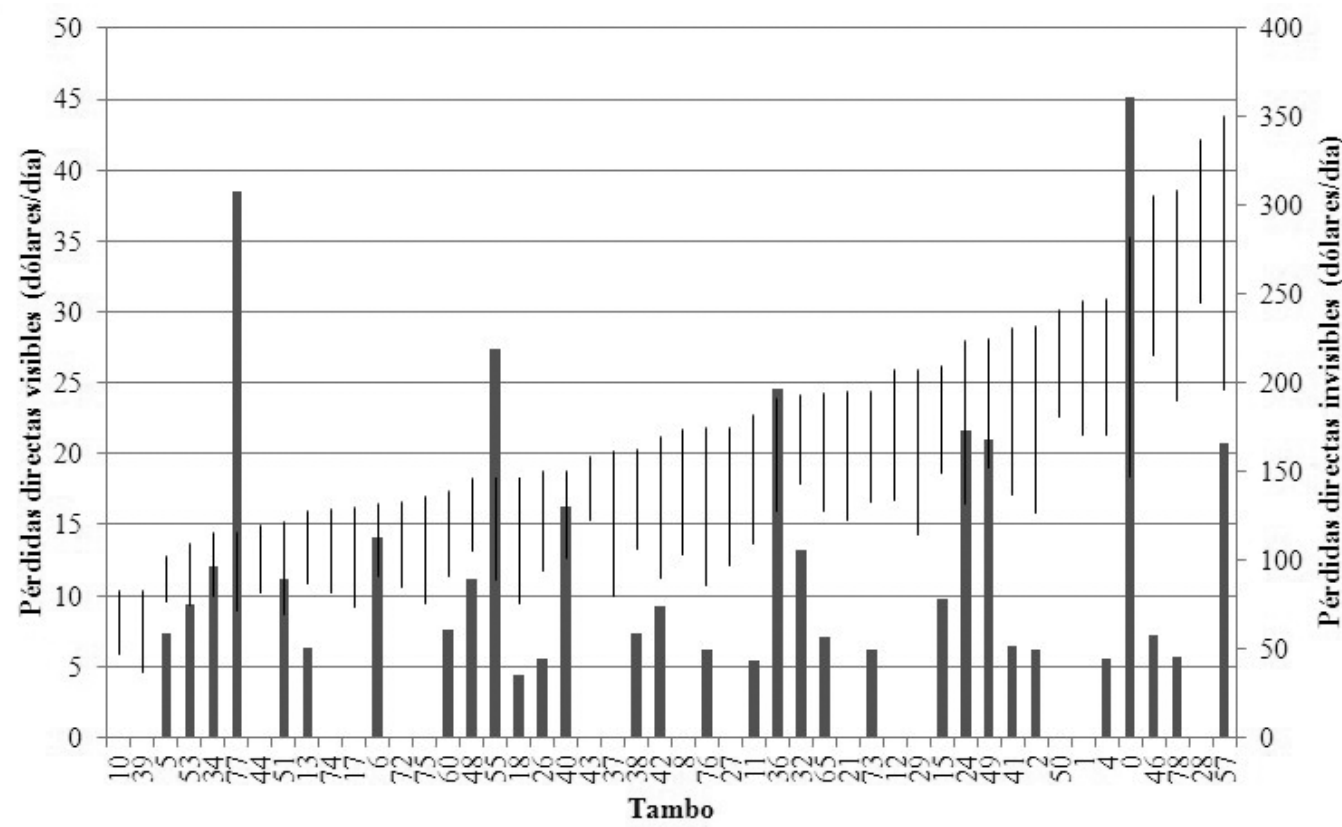

Figura 1. Pérdidas económicas por mastitis clínica y subclínica en establecimientos lecheros de Córdoba, Argentina $(\mathrm{n}=48)$.

Subclinical and clinical mastitis losses in dairy farms from Córdoba, Argentina $(n=48)$.

Barra vertical gris: total de pérdidas atribuidas a mastitis clínica en cada establecimiento; líneas verticales negras: pérdidas estimadas atribuidas a mastitis subclínica en cada establecimiento.

fue de US $\$ 0,059 /$ vaca/día (percentil $25 \%=0,034$; percentil $75 \%=0,095)$. Dentro de estas la erogación más importante fue por la terapia al secado, seguida por la del tratamiento antibiótico de los casos de MC (cuadro 2).

\section{ESTIMACIÓN DE LOS COSTOS TOTALES DE MASTITIS E IMPORTANCIA RELATIVA DE CADA COMPONENTE}

En el 50\% de los establecimientos estudiados el costo total fue $\geq$ US\$1,041/vaca/día, valor que ascendió a más de US\$1,203/vaca/día en aquellos establecimientos lecheros ubicados entre el $25 \%$ de los establecimientos con más altos costos totales por mastitis.

Al examinar la importancia relativa de los distintos componentes del costo respecto del total, se observó una gran variación entre establecimientos (figura 2). Las pérdidas de leche asociadas a MS constituyeron el concepto más importante dentro del costo total de la enfermedad en todos los establecimientos (figura 2). La mediana de importancia relativa de MS y MC fue de $88,8 \%$ (percentil $25 \%=82,8$; percentil $75 \%=93,5$ ) y $4,7 \%$ (percentil $25 \%=0,0$; percentil $75 \%=8,7$ ), respectivamente.

Al expresar los costos totales de la mastitis como una proporción de los ingresos brutos se observó que en el $50 \%$ de los establecimientos estos representaban al menos el $16,2 \%$ (percentil $25 \%=14,0$; percentil $75 \%=20,9$ )
ESTRATOS DE NIVELES DE PRODUCCIÓN Y COSTO DE LA MASTITIS

Del análisis de los costos por MS y MC y las erogaciones por control y prevención por estratos de establecimientos de acuerdo con los niveles de producción según los percentiles $25 \%$ y $75 \%$ no se observó ninguna tendencia, debido a la superposición de los rangos intercuartiles (cuadro 3). Sin embargo, al examinar la relación entre el costo total de la mastitis y los ingresos brutos para los estratos detallados se identificó una tendencia; el costo total de la mastitis representó el 24,3\% (percentil 25\% = 18,7; percentil 75\% = 33,3 ), 16,0\% (percentil 25\% $=13,6$; percentil $75 \%=18,3$ ) y $14,7 \%$ (percentil $25 \%=12,4$; percentil $75 \%=15,6$ ) de los ingresos brutos, para los estratos de menor a mayor nivel de producción de leche, respectivamente (cuadro 3).

\section{DISCUSIÓN}

Las pérdidas económicas producidas por la mastitis devienen tanto de la valoración de la frecuencia, la severidad y la duración de la enfermedad como del nivel productivo del establecimiento (Petrovski 2006). Esto podría explicar por qué los costos de la mastitis varían sensiblemente entre los productores, y es esto quizás lo más remarcable en este estudio. Dicha disparidad entre establecimientos es de particular interés en el análisis 
VISSIO Y COL

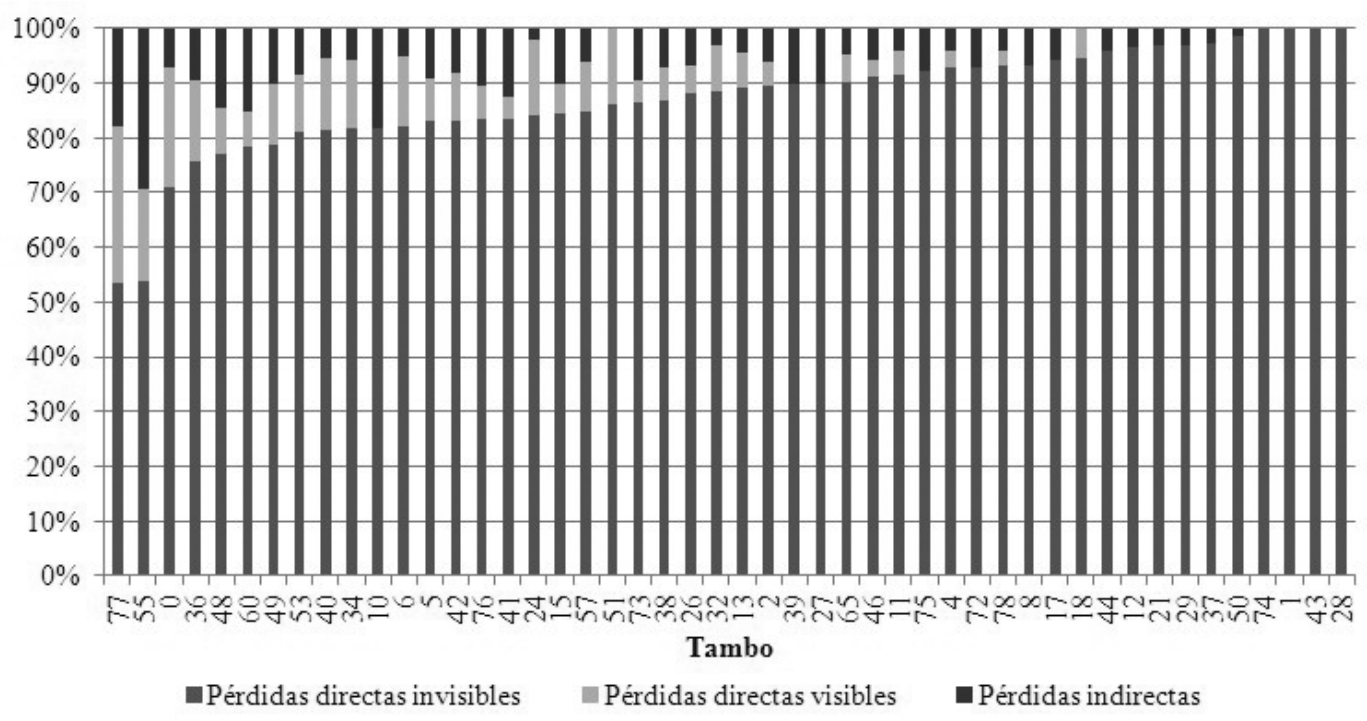

Figura 2. Importancia relativa de las pérdidas mastitis subclínica, clínica y de las erogaciones por aplicación de prácticas de control y prevención sobre el costo total de la enfermedad en establecimientos lecheros de Córdoba, Argentina $(\mathrm{n}=48)$.

Relative importance of the losses due to subclinical and clinical mastitis, and control and prevention expenditures over the total cost in dairy farms from Córdoba, Argentina $(n=48)$.

Barra vertical gris oscura: pérdidas económicas por mastitis subclínica; Barra vertical gris clara: pérdidas económicas por mastitis clínica; Barra vertical blanca: erogaciones por aplicación de prácticas de control y prevención asociadas a mastitis.

Cuadro 3. Costos de la mastitis (dólar/vaca/día) y su importancia relativa sobre los ingresos brutos diarios dentro de cada estrato de producción en establecimientos lecheros de Córdoba, Argentina $(\mathrm{n}=48)$. Argentina $(\mathrm{n}=48)$.

Mastitis costs (US\$/cow/day) y their relative weight over daily gross incomes within each production strata in dairy herds from Córdoba,

\begin{tabular}{|c|c|c|c|c|c|c|}
\hline & \multicolumn{6}{|c|}{ Estrato de producción } \\
\hline & \multicolumn{2}{|c|}{ <1.675 litros/día $(\mathrm{n}=12)$} & \multicolumn{2}{|c|}{$1.675-2.825$ litros/día $(n=24)$} & \multicolumn{2}{|c|}{$>2.825$ litros/día $(\mathrm{n}=12)$} \\
\hline & Costo & $\begin{array}{c}\% \text { Ingresos } \\
\text { brutos }\end{array}$ & Costo & $\begin{array}{c}\% \text { Ingresos } \\
\text { brutos }\end{array}$ & Costo & $\begin{array}{c}\% \text { Ingresos } \\
\text { brutos }\end{array}$ \\
\hline & \multicolumn{6}{|c|}{ Mediana (rango intercuartil) } \\
\hline MS & $\begin{array}{c}1,028 \\
(0,803-1,128)\end{array}$ & $\begin{array}{c}20,1 \\
(16,5-28,9)\end{array}$ & $\begin{array}{c}0,930 \\
(0,703-1,013)\end{array}$ & $\begin{array}{c}14,3 \\
(11,6-18,0)\end{array}$ & $\begin{array}{c}0,785 \\
(0,636-0,916)\end{array}$ & $\begin{array}{c}12 \\
(8,1-12,2)\end{array}$ \\
\hline $\mathrm{MC}$ & $\begin{array}{c}0,086 \\
(0,035-0,126)\end{array}$ & $\begin{array}{c}2,0 \\
(0,8-2,9)\end{array}$ & $\begin{array}{c}0 \\
(0-0,054)\end{array}$ & $\begin{array}{c}0 \\
(0-0,9)\end{array}$ & $\begin{array}{c}0,071 \\
(0,032-0,129)\end{array}$ & $\begin{array}{c}1,1 \\
(0,5-1,8)\end{array}$ \\
\hline $\begin{array}{l}\text { Erogaciones por } \\
\text { control y prevención }\end{array}$ & $\begin{array}{c}0,079 \\
(0,047-0,106)\end{array}$ & $\begin{array}{c}1,3 \\
(0,9-2,6)\end{array}$ & $\begin{array}{c}0,051 \\
(0,031-0,068)\end{array}$ & $\begin{array}{c}0,7 \\
(0,5-1,1)\end{array}$ & $\begin{array}{c}0,069 \\
(0,021-0,106)\end{array}$ & $\begin{array}{c}1 \\
(0,3-1,5)\end{array}$ \\
\hline Costo total & $\begin{array}{c}1,187 \\
(0,967-1,286)\end{array}$ & $\begin{array}{c}24,3 \\
(18,7-33,3)\end{array}$ & $\begin{array}{c}1,018 \\
(0,757-1,134)\end{array}$ & $\begin{array}{c}16,0 \\
(13,6-18,3)\end{array}$ & $\begin{array}{c}1,037 \\
(0,836-1,134)\end{array}$ & $\begin{array}{c}14,7 \\
(12,4-15,6)\end{array}$ \\
\hline
\end{tabular}

económico, y en este sentido, de mayor importancia que las estimaciones promedio (Hogeveen y col 2011), ya que la variabilidad permitiría inferir la magnitud de las pérdidas evitables. Dicho aspecto es aún de mayor significación en este estudio porque se utilizó una muestra aleatoria, lo que implica una inferencia a un conjunto mayor de establecimientos lecheros.
El costo total diario promedio de la mastitis reportado en el presente trabajo $(0,99$ US\$/vaca/día) fue superior a las cifras publicadas por Huijps y col (2008) que ascendieron a 0,39 US\$/vaca/día, a las registradas por Hagnestam-Nielsen y Ǿstergaard (2009) de US\$ 0,46/ vaca/día, y a los US\$ 0,40/vaca/día reportados por Halasa y col $(2009$ b) (comparación realizada a moneda constante 
del año 2012). Asimismo, se destacan otras discrepancias relacionadas con la composición relativa de los costos. En este sentido, Huijps y col (2008) identificaron como los componentes del costo más importantes las pérdidas por MS y el descarte de vacas, mientras que HagnestamNielsen y Ǿstergaard (2009) y Halasa y col (2009b) los casos de MC y el descarte de vacas, respectivamente. En este estudio, el componente más importante correspondió a las pérdidas por MS, estas representaron en promedio el $87 \%$ del costo total. Este valor fue superior al $46 \%$ reportado por Huijps y col (2008), quienes consideraron en el costo total a otros componentes, como por ejemplo el descarte de vacas, que podrían explicar las diferencias.

Los costos de mastitis en este estudio representaron en promedio el $16 \%$ de los ingresos brutos. Si bien no hay precedentes en relación con este indicador, el trabajo de Wellenberg y col (2002) remarca que las pérdidas por mastitis pueden representar el $20 \%$ de la producción potencial. En este sentido, el porcentaje estimado de pérdidas en el presente estudio podría constituir una subestimación, debido a que se relacionan con el nivel real y no con el nivel potencial de ingresos brutos.

Las diferencias observadas en el costo total de la mastitis entre establecimientos estuvo influenciada sensiblemente por aquellos componentes asociados a las pérdidas productivas, primariamente, a la forma subclínica y, secundariamente, a la forma clínica de la enfermedad. Dichas pérdidas podrían deberse, como ha sido mencionado por McInnerney y col (1992), al grado de aplicación de medidas de control y prevención y a la eficacia de las mismas en los establecimientos. Considerando la magnitud de las erogaciones derivadas del control y prevención, su contribución a la disparidad en el costo total sería marginal. En relación con el grado de aplicación, casi un $20 \%$ de los productores solo utilizaba como medida de control el tratamiento de casos de MC y alrededor de 60\% no contemplaba la aplicación de desinfección postordeño de pezones, medida profiláctica de conocida eficacia en la reducción de los niveles de mastitis (Pankey y col 1984).

La estratificación de los establecimientos de acuerdo al nivel de producción no mostró patrón alguno entre segmentos respecto de los distintos componentes de costo de la enfermedad (expresado en US\$/vaca/día). Esto indicaría que el rango de producción diaria observada entre los productores estudiados no discrimina diferentes niveles de salud mamaria entre establecimientos. Un dato que sustenta esta explicación es que no se observaron diferencias en la distribución de RCS y en la proporción de MC entre los estratos estudiados (dato no mostrado). En contraste, al evaluar el impacto que tiene el costo total de la enfermedad sobre los ingresos brutos se observó que en establecimientos de menor escala productiva el impacto era más importante. Específicamente, la fórmula utilizada permite estimar las pérdidas por MS independientemente del nivel de producción (Dürr y col 2008); esto implicaría que si el nivel de producción es menor, el impacto porcentual sobre los ingresos brutos es mayor.

Debido a la naturaleza del estudio realizado no fue posible capturar la dinámica en el tiempo de las pérdidas por la enfermedad. En relación con los componentes de costo considerados, algunos aspectos abordados en otras investigaciones (Petrovski 2006, Halasa y col 2007, Geary y col 2012), como el descarte prematuro de vacas, la penalización por altos RCS en tanque, las erogaciones por labores extras asociadas a la enfermedad y los cambios composicionales de la leche, no fueron contemplados en el presente estudio. Resta evaluar en futuras investigaciones cuán eficiente es el control de la mastitis llevado a cabo y qué aspectos motivan al productor el implementar un programa de control eficiente y permanente en el tiempo.

Esta investigación constituye el primer reporte en Argentina de pérdidas directas y gastos de control y prevención diarios relacionados a la mastitis bovina, basado en una muestra aleatoria de productores lecheros de una de las principales cuencas del país. El costo total de esta enfermedad experimenta importantes cambios entre establecimientos, así como la importancia relativa de los costos directos y las erogaciones derivadas de su control y prevención. La MS se destaca como el componente más importante del costo total. Estos resultados contribuirían a una mejor percepción del impacto de la enfermedad y ciertamente podrían motivar a sectores públicos y privados a reforzar las acciones de control y prevención de la mastitis entre los productores lecheros.

\section{REFERENCIAS}

Ali AKA, GE Shook. 1980. An optimum transformation for somatic cell concentration in milk. J Dairy Sci 63, 487-490.

Asociación Argentina De Lucha Contra Mastitis. 1983. Estimación de las pérdidas en volumen de producción de leche provocadas por la mastitis bovina en la República Argentina. Comm Fed Lechería Arch Lechería 6, 73.

Bartlett PC, GY Miller, CR Anderson, JH Kirk. 1990. Milk production and somatic cell count in Michigan dairy herds. J Dairy Sci 73, 2794-2800.

Bedolla CC, MER Ponce de León. 2008. Pérdidas económicas ocasionadas por la mastitis bovina en la industria lechera. REDVET 9, 1-26.

Calvinho LF, L Tirante. 2005. Prevalencia de microorganismos patógenos de mastitis bovina y evolución del estado de salud de la glándula mamaria en Argentina en los últimos 25 años. Rev FAVE - Cs Vet 4, 29-40.

Castellanos A, LC Issaly, GM Iturrioz, M Mateos, JC Terán. 2009. Análisis de la cadena de la leche en Argentina. En: Instituto Nacional de Tecnología Agropecuaria (ed). Estudios socioeconómicos de los sistemas agroalimentarios y agroindustriales.BuenosAires, Argentina, Pp 24-26.

Dürr W, RI Cue, HG Monardes, J Moro-Méndez, KM Wade. 2008. Milk losses associated with somatic cell counts per breed, parity and stage of lactation in Canadian dairy cattle. Livest Sci 117, 225-232.

Geary U, N López-Villalobos, N Begley, F McCoy, B O’Brien, L O'Grady, L Shalloo. 2012. Estimating the effect of mastitis on the profitability of Irish dairy farms. J Dairy Sci 95, 3662-3673.

González RN, JA Giraudo, JJ Busso, R Heredia. 1977. Investigación en mastitis subclínicas. I. Pérdidas económicas. Rev Med Vet 58, 431-438. 
Graaf T, RH Dwinger. 1996. Estimation of milk production losses due to sub-clinical mastitis in dairy cattle in Costa Rica. Prev Vet Med 26, 215-222.

Hagnestam-Nielsen C, U Emanuelson, B Berglund, E Strandberg. 2009. Relationship between somatic cell count and milk yield in different stages of lactation. J Dairy Sci 92, 3124-3133.

Hagnestam-Nielsen C, S Ǿstergaard. 2009. Economic impact of clinical mastitis in a dairy herd assessed by stochastic simulation using different methods to model yield losses. Animal 3, 315-328.

Halasa T, K Huijps, O Ǿsterás, H Hogeveen. 2007. Economic effects of bovine mastitis and mastitis management: A review. Vet $Q 29,18-31$.

Halasa T, M Nielen, APW De Roos, R Van Hoorne, G de Jong, TJGM Lam, T van Werven, H Hogeveen. 2009a. Production loss due to new subclinical mastitis in Dutch dairy cows estimated with a test-day model. J Dairy Sci 92, 599-606.

Halasa T, M Nielen, T van Werven, H Hogeveen. 2009b. Stochastic bio-economic model of bovine intramammary infection. Livestock Science 124, 259-305.

Hogeveen H, K Huijps, JGM Lam. 2011. Economics aspects of mastitis: New developments. New Zeal Vet J 59, 16-23.

Huijps K, T Lam, H Hogeveen. 2008. Costs of mastitis: facts and perception. J Dairy Res 75, 113-120.

Huijps K, H Hogeveen, TJGM Lam, AGJM Oude Lansink. 2010. Costs and efficacy of management measures to improve udder health on Dutch dairy farms. J Dairy Sci 93, 115-124.

Lavon Y, E Ezra, G Leitner, D Wolfenson. 2011. Association of conception rate with pattern and level of somatic cell count elevation relative to time of insemination in dairy cows. J Dairy Sci 94, 4538-4545.

McInerney JP, KS Howe, JA Schepers. 1992. A framework for the economic analysis of disease in farm livestock. Prev Vet Med 13, 137-154

Miller RH, PC Bartlett, SEE Lance, J Anderson, LE Heider. 1993a . Costs of clinical mastitis and mastitis prevention in dairy herds. J Am Vet Med Assoc 202, 1230-1236.

Miller RH, MJ Paape, LA Fulton, MM Schutz. 1993b. The relationship of milk somatic cell count to milk yields for Holstein heifers after first calving. J Dairy Sci 76, 728-733.

NMC, National Mastitis Council. 2003. Current Concepts of Bovine Mastitis. In: National Mastitis Council. $4^{\text {th }} \mathrm{ed}$. WD Hoard and Sons Co, Wisconsin, USA, Pp 1-64.
Pankey JW, RJ Eberhart, AL Cumming, RD Daggett, RJ Farnsworth, CK McDuff. 1984. Uptake on postmilking teat antisepsis. J Dairy Sci 67, 1336-1353.

Pech-Martínez VC, M Carvajal-Hernández, R Montes-Pérez. 2007. Impacto económico de la mastitis subclínica en hatos bovinos de doble propósito de la zona centro del estado de Yucatán. Trop Subtrop Agroecosyst 7, 127-131.

Petrovski KR, M Trajcev, GJS Buneski. 2006. A review of the factors affecting the costs of bovine mastitis. Afr Vet Assoc 77, 52-60.

Reneau JK. 2001. Somatic cell counts: Measures of farm management and milk quality. Proc National Mastitis Council, Reno, Nevada, USA, Pp 29-37.

Romero AT. 2004. Situación actual de la mastitis en México. Departamento Producción Animal, Facultad de Medicina Veterinaria y Zootecnia-Universidad Nacional Autónoma de México. México DF, Pp 122-134.

Rushton J. 2009. The economics of Animal Health \& Production. CABI Wallingford,UK.

Schepers JA, AA Dijkhuizen. 1991. The economics of mastitis and mastitis control in dairy cattle-a critical analysis of estimates published since 1970. Prev Vet Med 10, 213-224.

Seegers H, C Fourichon, F Beaudeau. 2003. Production effects related to mastitis and mastitis economics in dairy cattle herds. Vet Res 34, 475-491.

Thrusfield M. 2007. Veterinary Epidemiology. Blackwell Science. $3^{\text {rd }}$ ed. Oxford, USA, Pp 1-593.

Van Asseldonk MAPM, RJ Renes, TJGM Lam, H Hogeveen. 2010. Awareness and perceived value of economic information in controlling somatic cell count. Vet Rec 166, 263-267.

Vissio C, SA Dieser, CG Raspanti, JA Giraudo, CI Bogni, LM Odierno, AJ Larriestra. 2013. Dairy herd mastitis program in Argentina: farm clusters and effects on bulk milk somatic cell counts. Pak Vet $J$ 33, 80-84

Wellenberg GJ, WHM van der Poel, JT Van Oirschot. 2002.Viral infections and bovine mastitis: a review. Vet Microbiol 2361, 2-21.

Xunta de Galicia, OPS. 2006. EPIDAT 3.1. A Coruña, Xunta de Galicia, OPS, Washington, D.C., USA.

Yalcin C. 2000. Cost of mastitis in Scottish Dairy Herds with low and high subclinical mastitis problem. Tur J Vet Anim Sci 24, 465-472. 